\title{
Análisis de la adherencia de los urólogos españoles a las recomendaciones de diagnóstico y tratamiento de las guías de práctica clínica sobre incontinencia urinaria
}

\author{
Ruiz Cerdá JL, Arlandis Gúzman S, Trassierra Villa M, García Fadrique G, Morales Solchaga G, \\ Jiménez Cruz JF.
}

Servicio de Urología. Hospital Universitario La Fe. Valencia.

Actas Urol Esp. 2007;31(10):1148-1160

\begin{abstract}
RESUMEN
ANÁLISIS DE LA ADHERENCIA DE LOS URÓLOGOS ESPAÑOLES A LAS RECOMENDACIONES DE DIAGNÓSTICO Y TRATAMIENTO DE LAS GUÍAS DE PRÁCTICA CLÍNICA SOBRE INCONTINENCIA URINARIA

Objetivo: Ante la carencia de datos sobre el grado de adherencia a las guías clínicas sobre incontinencia urinaria, el objetivo fue conocer cómo la diagnostican y tratan los urólogos españoles.

Material y métodos: Sondeo de opinión quasi nacional sobre 202 urólogos en 20 reuniones representativas. Se realizaron 2 preguntas de diagnóstico y 2 de tratamiento, agrupando las respuestas por concepto. Se uso el método Grupo Focal dinamizado con "brain storming estructurado". La adherencia se estimó por la concordancia entre lo recomendado y lo practicado. Los resultados se promediaron según el total de respuestas.

Resultados: La adherencia fue: anamnesis $88 \%$, exploración física $78 \%$, analítica $47 \%$, sedimento $72 \%$ y cultivo $67 \%$. Para la urodinámica fue del $23 \%$ y para el uso de cuestionarios de síntomas y calidad de vida fue minoritaria con $14 \%$ y $26 \%$. La adherencia a preguntas correctas sobre el tipo de incontinencia fue muy elevada, necesitándose sólo 2,7 preguntas de media para el diagnóstico correcto. En el tratamiento de la incontinencia mixta, un 85\% abordó primero la urgencia y un $11 \%$ el esfuerzo. El 27\% considera que el principal motivo es la disponibilidad de drogas. Para el tratamiento de la incontinencia de urgencia, la adherencia al uso de antimuscarinicos fue del $98 \%$ y la de la modificación de hábitos del $50 \%$. En la incontinencia de esfuerzo, la adherencia en el uso de ejercicios del suelo pélvico fue del $81 \%$ y para la cirugía del $53 \%$.

Conclusiones: La adherencia de los urólogos españoles a las guías de IU está lejos de lo ideal. Aún con sesgos, los resultados pueden considerarse representativos. Es deseable que, en la creación de guías, se diseñen indicadores que midan el grado de adherencia.
\end{abstract}

Palabras clave: Adherencia. Guía clínica. Incontinencia urinaria.

\section{ABSTRACT}

ANALYSIS OF THE SPANISH UROLOGISTS ADHERENCE TO THE RECOMENDATIONS OF THE GUIDELINES ON DIAGNOSTIC AND TREATMENT OF URINARY INCONTINENCE

Objetive: Data regarding the degree of adherence to urinary incontinence guidelines are not available. The aim of this study was to know how the spanish urologist diagnos and treat urinary incontinence.

Material and methods: This is an opinion poll from 202 urologist underwent in 20 representative meetings of the Spain. The poll consisted of 2 questions about diagnostic and 2 about treatment of urinary incontinence. The anwsers were grouped by ideas. The focal group method dinamized by "structured brain storming" was used. We evaluate the adherence to the incontinence guide lines analizing the agreement between the recommendations and the real practice. We average acording all obtained responses.

Results: The adherence were: history $88 \%$, physical examination $78 \%$, blood test $47 \%$, urunalysis $72 \%$ and urine culture $67 \%$. The adherence for urodynamic investigation was $23 \%$. The use of symptom and quality of life cuestionares was minority (14\% y 26\%). The adherence to the right questions about the type of incontinece was very high. An average of 2.7 questions to reach correct diagnoses was needed. A $85 \%$ of urologist treat urge compenent of mixed incontinence firstly and $11 \%$ the stress component. The $27 \%$ considered the availability of drug as the main reason for such prioritization. The adherence of treating urge incontinence with antimuscarics was $98 \%$ and of life style interventions $50 \%$. For treating stress urinary incontinence, the adherence to pelvic floor muscle training was $81 \%$ and to surgery $53 \%$.

Conclusions: Spanish urologist adherence to incontinence urinary guidelines is far from the ideal. Although this study may have bias, the outcome can be considered as representative of the situation. It is desirable that, in the process of guidelines creation, specific parameters to assess the degree of adherence be designed.

Keywords: Adherence. Guidelines. Urinary incontinence. 
$\mathrm{E}$ las dos últimas décadas se ha extendido el uso de la guías de práctica clínica como una herramienta actual de medicina basada en la evidencia. Una guía clínica se define como los acuerdos desarrollados de forma sistematizada para asistir la decisión de los médicos y sus pacientes sobre el cuidado apropiado en circunstancias clínicas específicas. Son el producto del consenso sobre un proceso clínico mediante un método que garantiza su validez. Las recomendaciones no son vinculantes. Su existencia se justifica por la necesidad de homogeneizar la práctica clínica ante la amplia variabilidad existente. Además, sirven para disminuir el coste sanitario, evitar una asistencia inapropiada al paciente (infrautilización o sobreutilización de pruebas diagnósticas o de tratamiento), favorecer la traslación rápida de los avances científicos y estandarizar los procesos clínicos para su evaluación. Una guía debe ser clara, reproducible, consistente y de origen multidisciplinario.

La aplicabilidad de una guía en la práctica clínica diaria es un aspecto esencial. Porque, de su implantación efectiva, deriva la mejora en la calidad asistencial. Sin embargo, el mero hecho de realizarla y publicarla no significa que se aplique. Es decir, no es autoimplantable. Por ello, el concepto de guía debe ir ligado ineludiblemente al de evaluación de su aplicación. El proceso de evaluación conlleva siempre un ejercicio de autocrítica por parte de los profesionales. Ya que, detecta los posibles errores e identifica las áreas de mejora.

La incontinencia urinaria (IU) constituye un problema sanitario por su elevada prevalencia, gran impacto sobre la calidad de vida y elevado consumo de recursos económicos ${ }^{1-3}$. En la actualidad, se ha convertido en una enfermedad atractiva para la industria farmacéutica y para la ingeniería técnica sanitaria, por su gran beneficio económico potencial. Constantemente, se investigan nuevas modalidades terapéuticas farmacéuticas o instrumentales para la incontinencia urinaria de esfuerzo (IUE) y para la incontinencia urinaria de urgencia (IUU). Este desarrollo terapéutico ha llevado parejo un aumento de los profesionales sanitarios implicados en su manejo.
Los organismos internacionales de expertos y asociaciones científicas han diseñado guías clínicas de actuación diagnóstica y terapéutica para IU. Las dos guias más influyentes en nuestro ámbito científico son la de la International Consultation on Incontinence (ICI), formada por comités de expertos pertenecientes a la International Continente Society (ICS) y a la Societé Internationale d'Urologie (SIU), y la guía de la European Association of Urology (AEU) ${ }^{4,5}$. En nuestro país, se realizó una guía promovida por el Consejo de Salud Vesical en el año 2001. Sin embargo, no se ha vuelto a editar ni a revisar y sus contenidos son prácticamente iguales al de las otras $\operatorname{dos}^{6}$. En estas guías no se hace referencia a indicadores o estudios diseñados para evaluar su grado de cumplimiento. No se dispone de datos reales sobre si los profesionales implicados en el diagnostico y tratamiento de la IU están siguiendo sus recomendaciones. La ausencia de este feedback, de alguna manera, cuestiona la utilidad de las guías. Porque, sin datos que confirmen que se está realizando lo que la evidencia científica ha demostrado como beneficioso, no es posible mejorar.

Para enfrentarnos a esta carencia de información, la forma más sencilla de acercarse a la realidad es preguntar directamente a los profesionales sobre cuál es su práctica clínica habitual. El objetivo principal de nuestro estudio ha sido obtener información, lo más fiable posible, sobre cómo diagnostican y tratan la IU los urólogos españoles en su práctica diaria. Todo ello, para conocer el grado de adherencia y cumplimiento de las recomendaciones contenidas en las guías clínicas.

\section{MATERIAL Y MÉTODO}

Descripción del estudio

Se trata de un sondeo de opinión de ámbito quasi nacional sobre como se lleva a cabo el diagnóstico y tratamiento de la IU.

\section{Descripción de la muestra}

Entre los meses de octubre a noviembre del año 2005 se realizaron un total de 20 reuniones distribuidas por el territorio español (Fig. 1). Se consultó a 202 urólogos sin realizarse un cálculo previo del número que debía participar. Tampoco 


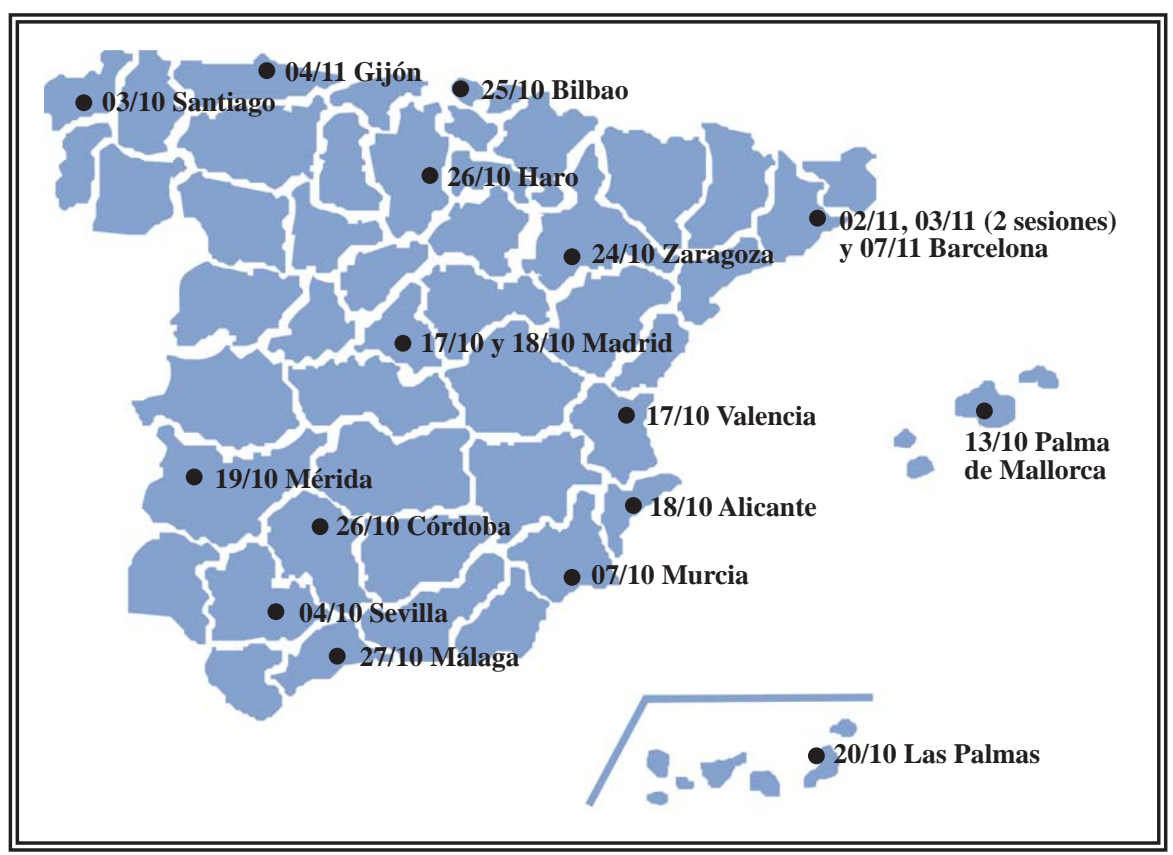

FIGURA 1.- Distribución por ciudades del territorio español, fechas y número de sesiones por ciudad de las 20 sesiones llevadas a cabo para estudio.

ideas aportadas por el conjunto del grupo, se comunican a éste por medio de un panel y a través del moderador.

\section{Formato de las reuniones}

Los participantes fueron invitados a participar en las reuniones. Previo al inicio de cada sesión, se realizó una introducción detallada de la metodología "brain storming estructurado" y se explicaron las normas a utilizar para el desarrollo de la misma. Los asistentes fueron distribuidos en una mesa abierta en forma de U con un panel dispuesto en frente para su correcta visión. Inicialmente, en los paneles únicamente se encontraba la pregunta a formular. Posterior-

se estableció ningún criterio específico de selección. Sin embargo, para obtener la mayor representatividad, la muestra consta de facultativos de todas las edades, diferentes periodos de tiempo ejerciendo la especialidad y desarrollo de actividad profesional y tanto de hospitales como de centros de salud.

\section{Metodología de estudio}

La técnica de recogida de información cualitativa se desarrolló mediante la metodología genérica denominada "Focus Group" o Grupo Focal. Se trata de un grupo de discusión alrededor de una pregunta concreta con el objetivo de recopilar información relativa a la misma ${ }^{7}$. Los grupos focales desarrollados se dinamizaron mediante la técnica de "brain storming estructurado". Esta técnica contempla una secuencia cerrada de los contenidos a discutir, cuyo tiempo y ordenación controla un moderador. La discusión de los contenidos se lleva a cabo a partir de una interacción regulada de los participantes con las diferentes agrupaciones de ideas que surgen del grupo a partir de una "pregunta llamada" u objeto de discusión planteado por el moderador. El método de aportación de información es anónimo. Los participantes no discuten entre ellos. Con todas las mente, los participantes emitian sus respuestas en tarjetas diseñadas específicamente para tal fin. Cada participante podía dar más de una respuesta. Tras la recogida de las tarjetas, todas las respuestas escritas fueron colocadas y ordenadas en el panel. Las respuestas de igual sentido fueron agrupadas y consideradas como una misma aportación individual. Finalmente, el contenido del panel era un reflejo fiel de la opinión global de los participantes con respecto a la pregunta planteada.

\section{Preguntas}

Se llevaron a cabo un total de 4 preguntas relacionadas con la IU. Dos estaban relacionadas con el diagnóstico (pregunta D1 y pregunta D2) y dos con el tratamiento (pregunta T1, y pregunta T2). En la Figura 2 se muestra el formato utilizado para cada pregunta. La pregunta D1 se llevó a cabo para estimar las diferencias entre la situación teórica de utilización de pruebas complementarias para el diagnóstico del tipo de IU (las recomendadas por las guías) y la realidad práctica (las que el urólogo lleva a cabo en la práctica clínica). En ella, se describían en una lista cerrada las pruebas utilizadas en el diagnostico de la IU. Esta pregunta poseía una segunda dimensión 


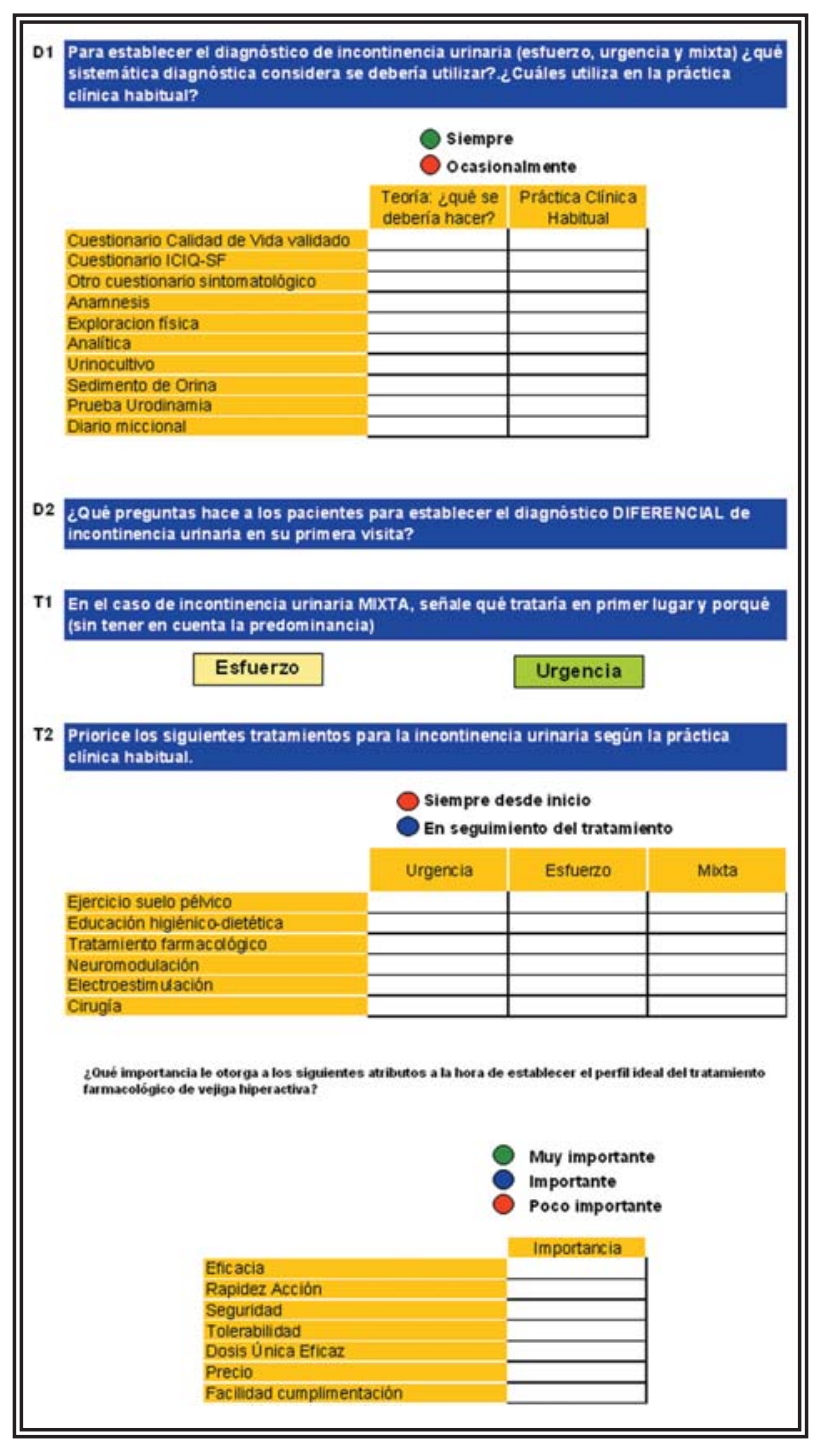

FIGURA 2.- Formato uniformizado del formulario empleado en las reuniones para la realización de las preguntas en cada una de las sesiones.

en la que se preguntaba, si la prueba debía realizarse siempre o de manera ocasional. Esta división trataba de identificar si la falta de cumplimiento era consecuencia de la ausencia de conocimiento y/o por falta de recursos para llevarlas a cabo. La utilización de cuestionarios de síntomas y de calidad de vida en la evaluación inicial de la IU está recomendada por la ICS ${ }^{5}$. Por lo que, también se recogió información sobre su uso. Entre las respuestas se incluyeron el uso de: cuestionario de vida validado, cuestionario mixto de sintomas, calidad de vida abreviado CIQ-SF y cualquier otro cuestionario usado. El diario miccional está considerado como la exploración fun- cional más básica. Informa sobre la frecuencia y volumen miccional. Estos parámetros se relacionan con la capacidad vesical. Aunque está altamente recomendado en las guías, no se tiene información sobre su uso en la práctica clínica ${ }^{4-5}$. Por lo que, también se incluyó como opción de respuesta.

La pregunta D2 era abierta y sin límite del número de posibles respuestas. Los urólogos tenían que indicar qué preguntas realizaban para diferenciar la IUE de la IUU de forma clínica.

La pregunta $\mathrm{T} 1$ era abierta y respondía a dos preguntas. La primera consistía en determinar que componente de la IU mixta (IUM), el de esfuerzo o el de urgencia, trataban primero. La segunda perseguía conocer los argumentos a favor de dicha priorización. Tampoco aquí se fijo límite al número de motivos, por lo que, las respuestas podían ser múltiples.

La pregunta T2 se llevó a cabo para conocer el tipo de tratamiento específico indicado en la IUE, IUU e IUM. La pregunta era cerrada con una lista de alternativas. Además, en la respuesta, se debía especificar si la terapéutica era de primera línea o sólo se reservaba para un segundo escalón durante el seguimiento del paciente. Todo ello, intentando ajustar las respuestas a las opciones de manejo inicial y especializado recomendado por las guías. Además, como parte de esta pregunta, se pidió que los urólogos definieran las características de lo que ellos consideraban antimuscarínico ideal.

\section{Análisis estadístico}

Los resultados obtenidos de las diferentes preguntas fueron agrupados según concepto y promediados en función del total de respuestas obtenidas. La adherencia se ha estimado por el grado de concordancia entre lo recomendado por las guías y lo realizado por los urólogos en la práctica clínica diaria. Los resultados se expresan en porcentajes.

\section{RESULTADOS}

Los resultados a la pregunta D1 se exponen en la Tabla 1. Existen diferencias entre lo que se considera debería hacerse y lo que realmente se hace en la práctica habitual. Las mayores diferencias se observan en la utilización del diario 
Tabla 1. Resultados obtenidos a la pregunta\&: ¿para establecer el diagnóstico de IU (esfuerzo, urgencia y mixta), ¿qué sistemática diagnóstica considera que se debería utilizar?

\begin{tabular}{|c|c|c|c|c|}
\hline \multirow{2}{*}{ Prueba diagnóstica } & \multicolumn{2}{|c|}{ Teoria (\%)* } & \multicolumn{2}{|c|}{ Práctica (\%)* } \\
\hline & Siempre & A veces & Siempre & A veces \\
\hline Anamnesis & 95 & 0 & 88 & 13 \\
\hline Exploración física & 93 & 1 & 78 & 15 \\
\hline Urocultivo & 74 & 16 & 67 & 20 \\
\hline Sedimento de orina & 74 & 9 & 72 & 13 \\
\hline Diario miccional & 63 & 30 & 25 & 45 \\
\hline Analítica & 46 & 26 & 47 & 26 \\
\hline Prueba urodinámica & 37 & 44 & 23 & 58 \\
\hline Cuestionario de calidad de vida & 26 & 42 & 12 & 34 \\
\hline Cuestionario ICIQ-SF & 14 & 44 & 6 & 33 \\
\hline Otro cuestionario sintomatológico & 11 & 35 & 8 & 34 \\
\hline
\end{tabular}

${ }^{\&}$ Los participantes valoraban, mediante un sistema de votaciones consistente en pegar adhesivos de color en la casilla elegida, con qué frecuencia se emplea cada técnica (verde = siempre, rojo = ocasionalmente). Se observan las diferencias entre uso teórico y práctico.

*Porcentaje que expresa el grado de consenso. Es la proporción de participantes que coincidio en la respuesta. El cálculo del \% se realizó sobre 202 participantes y las respuestas son independientes, por lo que la suma de los parciales no da como resultado 100 .

miccional (63\% utilización teórica versus 25\% utilización real) y en la exploración física $(93 \%$ utilización teórica versus $78 \%$ utilización real). En general, la anamnesis es el procedimiento más utilizado por los urólogos españoles para llegar al diagnóstico de la IU. Si bien, aunque en teoría debería utilizarse en todos los casos, en la práctica clínica no siempre es así. Por otra parte, aunque la adherencia al uso del sedimento de orina y la analítica no es completa, son los procedimientos con mayor consistencia teoría-práctica. Sólo se observan diferencias del $2 \%$ y $1 \%$ respectivamente. En cuanto al uso de la urodinámica en la sistemática diagnóstica, existen discrepancias de menor grado pero relevantes. Sólo el 37\% de los urólogos consideran que la prueba debería siempre realizarse. Sin embargo, en la práctica el porcentaje desciende al $23 \%$. El uso de los cuestionarios de síntomas y calidad de vida es minoritario. Tanto desde el punto de vista teórico como práctico e independientemente del tipo utilizado.

Las respuestas más representativas a la pregunta D2 se exponen resumidas en la Tabla 2. Se obtuvieron un total de 547 tarjetas. Fueron agrupadas por similitud de contenido dando lugar a un total de 16 ideas diferentes. Lo que supone que, los urólogos participantes necesitan un promedio de 2,7 preguntas para realizar el diagnóstico diferencial de IU. Una vez agrupadas según coincidencias de respuesta, se observó que las tres respuestas con mayor número de tarjetas fueron: ¿tiene incontinencia tras un deseo imperioso de orinar?, ¿tiene escapes de orina cuando realiza esfuerzos (tos, risa...)? y, a mayor distancia, ¿cuál es su frecuencia miccional (día/noche)?. Posteriormente, a partir de estas respuestas, se realizó una priorización de las 2 preguntas consideradas como más imprescindibles. Se eligieron las dos primeras.

Las respuestas obtenidas para la primera de las preguntas referentes a tratamiento de la IU
Tabla 2. Resultados obtenidos a la pregunta ${ }^{\&}$ ¿¿ué preguntas hace a los pacientes para establecer el diagnóstico diferencial de incontinencia urinaria en su primera visita?

\begin{tabular}{|c|c|c|}
\hline Respuesta & $\mathbf{N}$ & $\begin{array}{c}\text { *Respuestas } \\
\text { acumuladas } \\
\%\end{array}$ \\
\hline $\begin{array}{l}\text { ¿Tiene usted incontinencia tras un } \\
\text { deseo imperioso de orinar? }\end{array}$ & 175 & 86,6 \\
\hline $\begin{array}{l}\text { ¿Tiene escapes de orina cuando } \\
\text { realiza esfuerzos (tos, risa...)? }\end{array}$ & 152 & 75,2 \\
\hline $\begin{array}{l}\text { ¿Cuál es su frecuencia miccional } \\
\text { (día/noche)? }\end{array}$ & 53 & 26,1 \\
\hline ¿Hay nicturia? & 30 & 14,8 \\
\hline $\begin{array}{l}\text { ¿Cuántas compresas se cambia } \\
\text { al dia?¿Cuánto se le escapa? }\end{array}$ & 30 & 14,8 \\
\hline ¿Tiene molestias al orinar? & 29 & 14,3 \\
\hline ¿Desde cuando se le escapa? & 11 & 5,6 \\
\hline ¿Se le escapa en la cama? & 9 & 4,6 \\
\hline ¿Se le escapa continuamente? & 9 & 4,6 \\
\hline ¿Con qué frecuencia se le escapa? & 5 & 2,4 \\
\hline ¿Cómo se le escapa? & 5 & 2,4 \\
\hline $\begin{array}{l}\text { ¿Incontinencia diurna, nocturna } \\
\text { o ambas? }\end{array}$ & 5 & 2,4 \\
\hline ¿Con qué relaciona las pérdidas? & 5 & 2,4 \\
\hline ¿Cuántos partos ha tenido? & 5 & 2,4 \\
\hline ¿Nota que se va a orinar? & 5 & 2,4 \\
\hline ¿Tiene antecedentes médicos & & \\
\hline relacionados? & 5 & 2,4 \\
\hline Otras preguntas & 13 & 6,4 \\
\hline
\end{tabular}

\&Pregunta abierta sin limite del número de respuestas (202 urólogos - 547 respuestas). Se trataba de recoger que preguntas se realizan a los pacientes para diagnosticar el tipo de IU. Las respuestas se expresaban en el panel con tarjetas ( 1 tarjeta - 1 respuesta). *Proporción de participantes que coincidió en la respuesta. Cada urólogo podía responder más de una opción, con lo que el total de porcentajes no tiene que sumar 100. 
se exponen en la Tabla 3. Del total de 202 urólogos consultados, 172 (85\%) tratarían en primer lugar la IUU, 23 (11\%) tratarian primero la IUE y 7 (3\%) ambas simultáneamente. El principal argumento por el que se trataría de manera prioritaria la IUU es la disponibilidad de tratamiento farmacológico (sencillo y rápido). Este motivo representó el 27\% del total de repuestas que priorizaban el tratamiento del componente de urgencia de la IUM. Otras de las respuestas obtenidas argumentando lo mismo fueron: mayor mejora de la calidad de vida (17\%), el tratamiento farmacológico es reversible y menos agresivo (13\%), mejora rápida de los síntomas (10\%), si mejora el componente de urgencia hay pacientes que no se operan del componente de esfuerzo o para ver cuál de los dos componentes predomina ( $8 \%$ en ambos casos), entre otras. Por otra parte, los principales argumentos obtenidos para tratar en primer lugar el componente de esfuerzo fueron: al corregir el esfuerzo se corrige un elevado porcentaje de urgencia (35\% de las aportaciones en este ámbito concreto), condiciona más la vida social (13\% de las aportaciones) o porqué opinan que es la principal causa de IUM (9\%) (Tabla 4).

Tabla 3. Motivos argumentados para justificar el tratamiento prioritario del componente de urgencia en la IUM

\begin{tabular}{|c|c|c|c|}
\hline Motivo & $\mathbf{N}$ & $8 \%$ & $* \%$ \\
\hline $\begin{array}{l}\text { Disponibilidad de tratamiento } \\
\text { farmacológico sencillo y rápido }\end{array}$ & 56 & 24,1 & 27,7 \\
\hline Mejora más la calidad de vida & 35 & 15,0 & 17,3 \\
\hline $\begin{array}{l}\text { El tratamiento farmacológico es } \\
\text { reversible o menos agresivo }\end{array}$ & 26 & 11,2 & 12,8 \\
\hline Mejora rápida de los sintomas & 21 & 9,0 & 10,4 \\
\hline $\begin{array}{l}\text { Si mejora el componente de } \\
\text { urgencia hay pacientes que no } \\
\text { se operan }\end{array}$ & 17 & 7,3 & 8,4 \\
\hline $\begin{array}{l}\text { Para ver cuál de los dos } \\
\text { componentes predomina }\end{array}$ & 16 & 6,9 & 7,9 \\
\hline $\begin{array}{l}\text { Mientras se prepara la intervención } \\
\text { quirúrgica }\end{array}$ & 6 & 2,5 & 2,9 \\
\hline $\begin{array}{l}\text { Al corregir urgencia a veces se } \\
\text { corrigen las dos }\end{array}$ & 6 & 2,5 & 2,9 \\
\hline $\begin{array}{l}\text { La intervención quirúrgica de } \\
\text { esfuerzo puede empeorar la de } \\
\text { urgencia }\end{array}$ & 5 & 2,1 & 2,4 \\
\hline Otras respuestas & 14 & 6,0 & 6,9 \\
\hline
\end{tabular}

\&Indica el \% de tarjetas con la misma idea sobre el total de los emitidos $(\mathrm{n}=232)$. Posibilidad de más de una respuesta.

*Indica el \% de tarjetas con la misma idea sobre la IUU ( $\mathrm{n}=202)$.
Tabla 4. Motivos argumentados para justificar el tratamiento prioritario del componente de esfuerzo en la IUM

\begin{tabular}{lccc}
\hline Motivo & N & \&\% & $* \%$ \\
\hline $\begin{array}{l}\text { Al corregir esfuerzo se corrige un } \\
\text { elevado porcentaje de urgencia }\end{array}$ & 8 & 3,4 & 34,7 \\
$\begin{array}{l}\text { Condiciona más la vida social } \\
\begin{array}{l}\text { Principal causa de incontinencia } \\
\text { urinaria mixta }\end{array}\end{array}$ & 3 & 1,2 & 13,0 \\
Otras respuestas & 2 & 0,8 & 8,70 \\
\hline
\end{tabular}

\&Indica el \% de tarjetas con la misma idea sobre el total de los emitidos $(\mathrm{n}=232)$.

*Indica el \% de tarjetas con la misma idea sobre la IUE $(n=23)$.

En la pregunta T2 sobre tratamiento de la IU, se priorizaron los tipos de tratamiento para cada una de las IU según su experiencia. Para la IUU (Fig. 3), el tratamiento farmacológico desde inicio fue la opción (98,0\%), seguida por la educación higiénico-dietética con un 50\% de los votos como tratamiento a instaurar desde el inicio y 6,9\% como tratamiento durante el seguimiento. Dentro de este apartado, se solicitó la opinión de los urólogos sobre el perfil del antimuscarínico ideal para el tratamiento de la IUU por vejiga hiperactiva. Según la opinión mayoritaria de los facultativos, este tratamiento debería tener una elevada eficacia, tolerabilidad y seguridad, como se muestra en los resultados de la Figura 4. Además, sería interesante que tuviera facilidad de cumplimiento por parte del paciente, que tuviera una acción rápida y dosis única eficaz. El precio del fármaco no fue valorado como muy importante en la toma de decisión del antimuscarínico ideal. La neuromodulación y la electroestimulación fueron las opciones terapéuticas de segunda línea más votadas con un $22,8 \%$ y $20,3 \%$ de votos, respectivamente.

Para el tratamiento de inicio de la IUE (Fig. 5) la opción más votada fue los ejercicios de suelo pélvico $(80,7 \%)$. La cirugía fue también considerada como opción terapéutica de inicio por el $51,2 \%$ de los especialistas. Aunque, fue también la primera opción a tener en cuenta como terapéutica de segunda línea, con un $48,8 \%$ de votos.

Para la IUM los urólogos consultados utilizaban mayoritariamente tratamientos farmacológicos desde inicio (Fig. 6). Además del tratamiento 


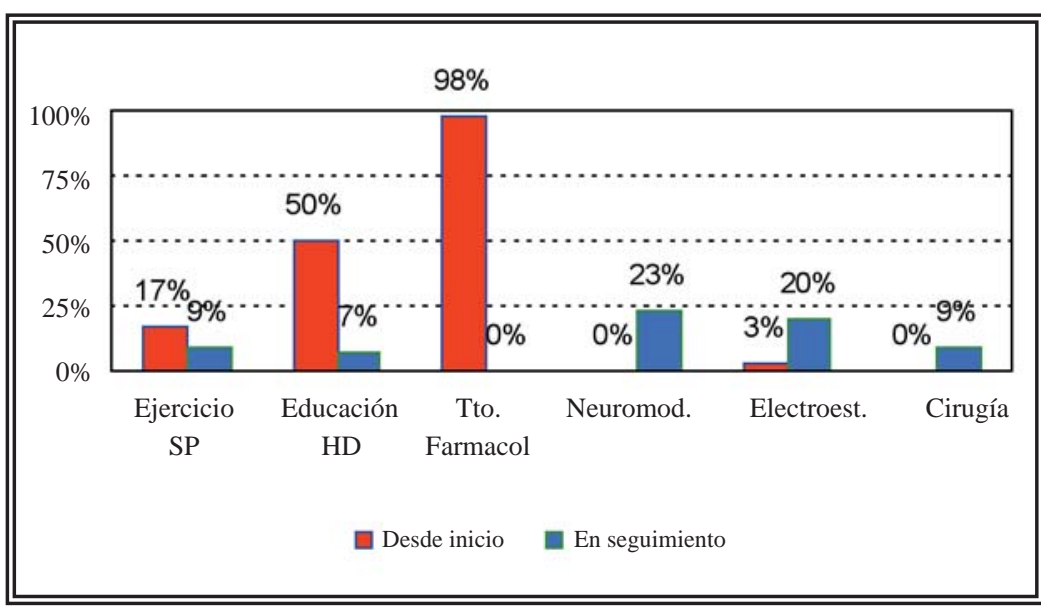

FIGURA 3.- Alternativas terapéuticas para la IUU. Los participantes vota ron sobre la opción elegida sobre el tipo de tratamiento y en qué momento evolutivo. Respuesta cerrada mediante la colocación de adhesivos de colores (rojo= desde inicio, azul= en el seguimiento).

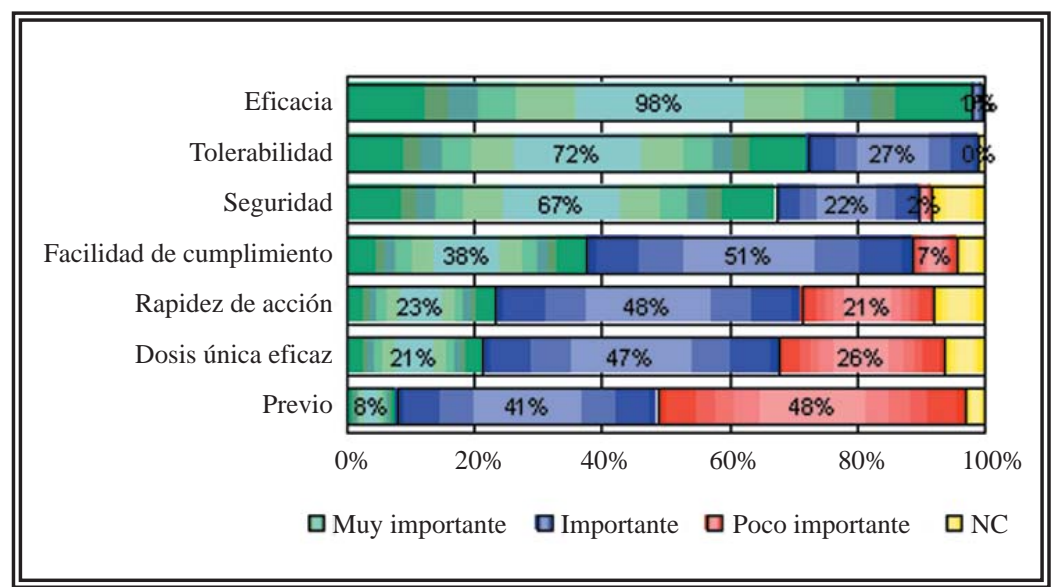

FIGURA 4. Resultados de las caracteristicas del perfil del antimuscarinico ideal para el tratamiento de la IUU según importancia para el urólogo.

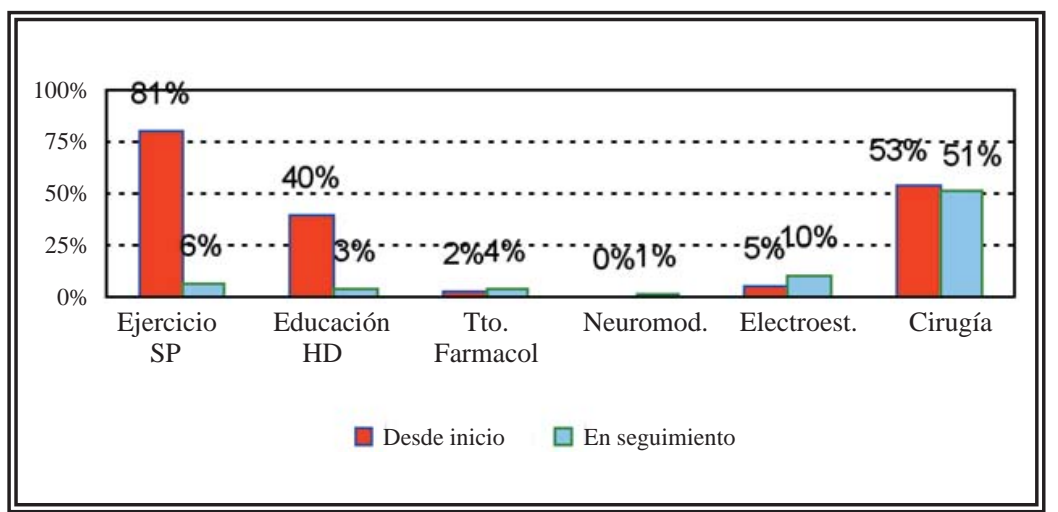

FIGURA 5. Alternativas terapéuticas para la IUE. Los participantes vota ron sobre la opción elegida sobre el tipo de tratamiento y en qué momento evolutivo. Respuesta cerrada mediante la colocación de adhesivos de colores (rojo= desde inicio, azul= en el seguimiento). farmacológico, casi la mitad de los participantes consideraron que los ejercicios de suelo pélvico y la educación higiénico-dietética eran también opciones de tratamiento inicial con el $48,5 \%$ y $40,1 \%$ de votos respectivamente. La cirugía fue la principal opción como terapia de segunda línea o de seguimiento con un $52,5 \%$ de votos.

\section{DISCUSIÓN}

El manejo diagnóstico de la IU se basa en un cuidadoso interrogatorio, exploración física abdominal y dirigida, analítica, exploraciones radiológicas y exploraciones funcionales urodinámicas más o menos invasivas. En casos complejos se necesitan exploraciones sofisticadas como la videourodinámica. Además, se recomienda la utilización de cuestionarios de calidad de vida. Ya que, mejoras en las variables de calidad de vida se correlacionan directamente con la eficacia terapéutica ${ }^{8}$. La sistemática de actuación diagnóstica varia dependiendo del ambiente asistencial donde trabaje el urólogo. El objetivo del diagnostico es evidenciar los escapes, observar sus características, cuantificar su severidad e identificar el mecanismo fisiopatológico. Este último aspecto es esencial para dirigir el tratamiento. Principalmente, porque la IUE tiene tratamiento predominantemente quirúrgico y la IUU, al menos inicialmente, médico. El diagnóstico diferencial entre estos tipos de IU es esencial. Las equivocaciones exponen a los pacientes a tratamientos inadecuados, o lo que es peor, a intervenciones inapropiadas con graves consecuencias en casos concretos. En ninguna otra enfermedad como la IU se cumple la máxima de que el mejor tratamiento es un buen diagnóstico. Ya que el resultado terapéutico depende de la indicación adecuada. 


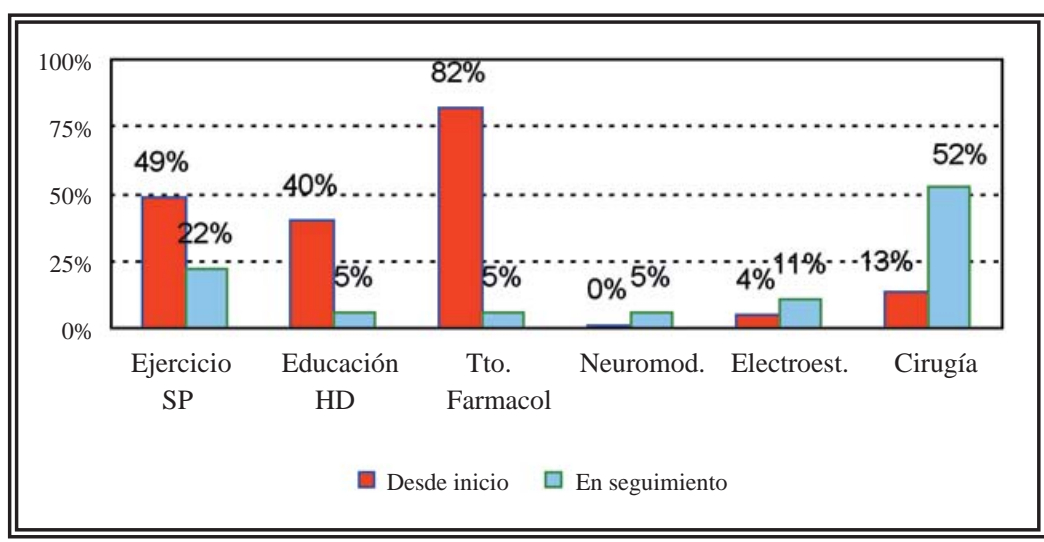

FIGURA 6.- Alternativas terapéuticas para la IUM. Los participantes vota ron sobre la opción elegida sobre el tipo de tratamiento y en qué momento evolutivo. Respuesta cerrada mediante la colocación de adhesivos de colo res (rojo= desde inicio, azul= en el seguimiento).

Aunque se ha avanzado en el conocimiento de la IU, sigue existiendo gran variabilidad en la sistemática diagnóstica y terapéutica utilizada entre profesionales. Puesto que, lo recomendado en las guías clínicas, desde un punto de vista teórico, no siempre es fácil llevarlo a la práctica. Actualmente, además de urólogos especializados, existe una gran diversidad de profesionales implicados en el manejo de la IU: urólogos generales, geriatras, ginecólogos, fisioterapeutas, médicos generales y enfermeras especializadas. Cada uno de ellos con diferente ambiente y logística de trabajo y con diferente grado de conocimiento sobre la IU. Es por esto que, conocer el grado de adherencia y cumplimiento de las recomendaciones de las guías clínicas es de gran importancia. Ya que, incide directamente sobre su razón de ser: mejorar la calidad del cuidado del paciente al reducir la variabilidad no justificada y difundir la aplicación de avances efectivos en la actividad clínica diaria.

Para mejorar es necesario medir, y lo que no es medible no es, por tanto, evaluable. Sin datos que nos confirmen que realizamos lo que la evidencia científica ha demostrado como beneficioso en nuestros procesos clínicos, será imposible mejorar. Es evidente que, todo el esfuerzo realizado por las organizaciones científicas y grupos de expertos para crear guías clínicas de IU resulta baldío si no se dispone de datos sobre el grado de cumplimiento real. Otras especialidades disponen de este tipo de datos pero en urología existe una carencia al respecto ${ }^{9}$. Tanto en las tres edi- ciones de la guía de la ICI (2001, 2003, 2005) como en todas las de la guía de la EAU (2001-2007), las modificaciones se han realizado por consenso de grupos de expertos tras una actualización de la evidencia científica. Todo ello sin evaluar el grado de cumplimiento real obtenido tras la publicación de las ediciones previas. El motivo ha sido que, en el diseño de las guías, no se tuvo en cuenta su evaluación. Aspecto esencial para poder favorecer su implantación. Puesto que, el feedback de su aplicación permite valorar, por un lado, su factibilidad $y$, por otro, las dificultades de su puesta en marcha.

Una vez se asume la necesidad de medir para evaluar, este proceso debe utilizar una metodología concreta. Es necesario construir indicadores específicos basados en las guías que permitan registrarlos y medirlos de forma sencilla. Estos indicadores deben presentar unas características específicas para que sean evaluables. Sobre la base de las guías se priorizan indicadores de calidad (por ejemplo, uso de antimuscarínicos en IU mixta en ausencia de contraindicación), y a partir de éstos se construyen unas medidas de cumplimiento (por ejemplo, porcentaje de pacientes tratados con antimuscarínicos sobre el total de pacientes con IU mixta sin contraindicaciones). Es evidente que existe una carencia de indicadores específicos que permitan estudiar este campo de la IU.

El objetivo de este estudio era analizar comparativamente la práctica clínica habitual de los urólogos españoles con respecto a las guías de práctica clínica existentes Si bien este estudio presenta carencias metodológicas, los resultados obtenidos a partir de las 20 sesiones pueden considerarse en gran medida representativos. Además, permiten un análisis cualitativo de la realidad asistencial actual sobre el diagnóstico y tratamiento de la IU. Para obtener la opinión de los urólogos participantes se utilizó el método de grupo focal mediante la dinamización de la técnica "brain storming" estructurado ${ }^{7}$. Esta técnica garantiza la aportación de ideas por igual de todos los participantes con un número mínimo y 
máximo de aportaciones. Por otro lado, el anonimato de las aportaciones de ideas minimiza las posibles distorsiones que puedan ocasionar la figura del "lider" o "protagonista" y los posibles casos de "cohibición" o reparos a expresar en público opiniones concretas. Por lo que, se evita la monopolización del debate por una o varias personas ${ }^{10}$.

Los enfoques de la guía de la ICI y la de la EUA son muy parecidos ${ }^{4,5}$. La primera clasifica las pruebas para la evaluación de la IU según la conveniencia de su utilización basada en la evidencia científica existente. Una prueba es altamente recomendable cuando se debería hacer a todos los pacientes. Una prueba es recomendable cuando se ha demostrado su valor en la mayoría de pacientes y se recomienda encarecidamente su uso en la evaluación inicial. Una prueba es opcional cuando se ha demostrado útil en la evaluación de determinados pacientes seleccionados. Por lo que, se deja al juicio del médico su utilización. Una prueba no es recomendada cuando no ha demostrado poseer ningún valor ${ }^{5}$. La guía de la EAU divide la actuación de acuerdo a diferentes grupos clínicos entre mujeres, hombres, neurológicos, ancianos y niños con IU. Además, en cada grupo clínico se subdivide la valoración y el tratamiento en inicial y especializado ${ }^{4}$. Este abordaje diagnóstico y terapéutico está condicionado por la discrepancia en el número de urólogos por habitantes existente entre países de Europa. En el norte europeo la densidad de urólogos es inferior a la de España o Italia. Esta circunstancia hace que la asistencia recaiga en gran medida en los médicos generales. Por lo tanto, de entrada, hay barreras que impiden su adecuada implantación en nuestro medio.

De acuerdo a las recomendaciones de la guía de la ICI, las pruebas altamente recomendables en la evaluación inicial de un paciente con IU son: historia clínica detallada, exploración física, sedimento de orina y cuantificación de síntomas $^{5}$. Según la guía de la EUA el manejo inicial debe constar de valoración sintomatológica, diario miccional, valoración de impacto sobre calidad de vida, examen físico, sedimento y cultivo y medición de residuo postmiccional. Además se resalta que, la exploración física debe incluir una prueba de esfuerzo con maniobra de valsalva o tos, que el sedimento es fundamental y necesario en todos los pacientes con IU y que la analítica sanguínea para valorar la función renal se recomienda sólo antes de cirugía ${ }^{4}$. La mayoría de urólogos coinciden en gran medida con lo recomendado. Si bien, existe una pequeña diferencia teórico-práctico. Hay que resaltar que, un 15\% sólo llevan a cabo la exploración física a veces, cuando con una prueba esfuerzo simple se llega al diagnostico de IUE no complicada. Incluso, este porcentaje puede ser mayor. Ya que, la prueba de esfuerzo requiere llenar la vejiga con $200 \mathrm{ml}$. Lo cual no es siempre posible en una consulta ambulatoria saturada de pacientes. En cuanto al sedimento y el cultivo de orina existe coincidencia teórico-práctico. Ambas pruebas son esenciales de acuerdo a las guías clínicas.

La historia clínica debe incluir una valoración de síntomas mediante un diario de frecuencia- volumen y un cuestionario sintomatológico ${ }^{4,5}$. El diario miccional es la prueba adicional más efectiva junto a la evaluación básica. Es una herramienta muy útil desde el punto de vista diagnóstico y funcional. Está considerada como la prueba urodinámica más básica. Ya que, aporta información sobre el volumen miccional, parámetro funcional esencial en el planteamiento terapéutico de cualquier tipo de IU. De acuerdo a los resultados obtenidos, su uso es realmente bajo. La mayoría de urólogos son conscientes de su utilidad, pero en la práctica sólo un $25 \%$ lo llevan a cabo. Las causas del escaso uso pueden ser varias. No hay un formato estandarizado, el grado de cumplimentación depende del nivel educativo, edad y situación social del paciente y su análisis requiere tiempo. Por otra parte, no resulta fácil realizar comparaciones entre la situación basal y post-tratamiento fuera del ámbito de ensayos clínicos. Todo ello refleja una realidad; que la información y el conocimiento se tienen, pero la implantación de esta herramienta está muy lejos de lo recomendado en las guías.

Básicamente, existen dos tipos de cuestionarios: los diagnósticos y los de calidad de vida específicos de enfermedad ${ }^{8}$. Los primeros evalúan los síntomas por si mismos. Se utilizan para detectar, diagnosticar y evaluar el efecto terapéutico. Los segundos evalúan los síntomas miccio- 
nales y su impacto sobre la calidad de vida. La ICI recomienda incluirlos en todos los estudios de IU como complemento a las medidas clínicas para medir el efecto terapéutico ${ }^{5}$. Ante la necesidad de comparar y por razones de investigación, ambos tipos de cuestionarios deben estandarizarse. El ICIQ-SF es un cuestionario mixto sintomatológico-calidad de vida desarrollado por la ICI y respaldado con un grado de evidencia que lo cataloga como altamente recomendable. Es sencillo, fiable, práctico y robusto, lo que ha facilitado su implantación. En la actualidad, es el más utilizado tanto en ensayos clínicos como en estudios epidemiológicos. Con tan sólo 6 preguntas, cuantifica la severidad de la IU, su repercusión sobre la calidad de vida y orienta sobre el tipo de IU. Es muy útil en la evaluación basal de pacientes con IU y tras tratamiento. Además, presenta elevada correlación con los parámetros urodiná$\operatorname{micos}^{12}$. Sin embargo, como demuestran los resultados de este estudio, la utilización de cualquier tipo de cuestionario sigue siendo minoritaria en la práctica clínica. Lo que realmente llama la atención es que, aunque existe discrepancia teórico-práctico, el porcentaje de urólogos que lo consideran necesario es realmente bajo. Por lo que, en este punto, refleja una gran distancia entre lo recomendado por las guías y el conocimiento que tienen los urólogos. Es una realidad que, en la práctica clínica y aparte de ensayos clínicos, los cuestionarios de calidad de vida no se usan. Las causas de la falta de implantación del uso de cuestionarios son: desconocimiento de su recomendación, consumo de tiempo para complementar, dificultad comparar entre situación basal y post-tratamiento y gran variabilidad en el grado de cumplimentación.

Aparte del diario miccional, el uso de pruebas urodinámicas en la evaluación de la IU es un tema controvertido. La flujometría y la medición de residuo postmiccional son pruebas funcionales no invasivas de detección de disfunción de vaciado. La cistomanometría de llenado y vaciado, la prueba de esfuerzo y el perfil uretral son pruebas invasivas por utilizar catéteres. Durante el llenado, informan sobre la sensación vesical, el comportamiento del detrusor y la competencia uretral. Durante el vaciado, informan sobre la contractilidad y la resistencia uretral. De acuerdo a las reco- mendaciones de la guía de la ICI, el diario miccional, la medición de residuo postmiccional y la flujometría estarian recomendadas en todos los pacientes con $\mathrm{IU}^{5}$. Sin embargo, no es necesaria la realización de pruebas invasivas antes del tratamiento cuando la IU no se asocia a factores que la hagan considerar complicada. Sobre todo, si se no va a realizar un tratamiento quirúrgico. Aquí entrarían las IUE e IUU puras sintomáticas no complicadas, sin sintomas ni signos de disfunción de vaciado. Por el contrario, las pruebas invasivas estarian recomendadas antes de cualquier tratamiento quirúrgico, después de fallo de tratamiento, cuando se necesita más información para planificar posteriores terapias, como parte del seguimiento de pacientes neurológicos y en IU complicadas. En estas circunstancias, se justifica para tener un conocimiento objetivo de la patología subyacente y realizar un tratamiento más racional. Por todo ello, en la guía de la EUA el estudio urodinámico sólo está recomendado a nivel especializado ${ }^{4}$. De acuerdo a los resultados obtenidos, el uso de pruebas de urodinámica para el diagnóstico de la IU es muy bajo. Tanto desde el punto de vista teórico como práctico. Tal y como se formuló la pregunta, la posible respuesta incluía tanto pruebas urodinámicas no invasivas, a excepción del diario miccional, como invasivas utilizadas en el diagnostico inicial de la IU. Por lo tanto, los resultados obtenidos se ajustan a lo recomendado por las guías. Sin embargo, el uso de pruebas funcionales está condicionado, además de por la invasividad, por la accesibilidad que tenga el urólogo en su ámbito de trabajo. Existen tres ámbitos de trabajo con acceso progresivo a las diferentes pruebas. En el primero se encuentran los urólogos de consultas en las que únicamente se dispone de historia clínica, exploración física, evaluación de síntomas y de diario miccional. En el segundo se encuentran los urólogos con acceso a pruebas urodinámicas no invasivas (ecografía vesical para medición de residuo postmiccional y flujometría). Por último, en el tercer nivel se encuentran los urólogos con acceso a estudio urodinámico completo del tracto urinario inferior. Por lo que, los resultados deben ser analizados con cautela. Ya que, se desconoce la accesibilidad de cada participante a la urodinámica. 
Aunque el valor individual de los síntomas urinarios para predecir la fisiopatología subyacente de la IU no es elevado, una importante proporción de mujeres con IU pueden ser correctamente diagnosticadas clínicamente. En la evaluación inicial de un paciente con IU se identifican tres grupos de pacientes: IUE pura, IUU, en el contexto de una vejiga hiperactiva, o IUM. Los porcentajes obtenidos en la pregunta dos de diagnóstico ponen de manifiesto que las preguntas realizadas para diferenciar entre IUE e IUU son adecuadas. Si bien, se mezclan con preguntas no propiamente diagnósticas. Como por ejemplo, de tipo de IU ¿cómo se le escapa?, evolutivas ¿desde cuando se le escapa? o de cuantificación de severidad ¿cuántas compresas usa?.

De acuerdo a la definición de la ICI, la IUM es un concepto clínico caracterizado por la percepción de pérdida involuntaria de orina asociada tanto a la urgencia como al esfuerzo ${ }^{12}$. Esta definición se presta a diversas interpretaciones y no esta clara. Se deja abierta la posibilidad de que ambos tipos de escapes se produzcan simultáneamente o no. Por otra parte, desde el punto de vista urodinámico, se refiere a la demostración de escapes relacionados con el aumento de presión intraabdominal y con la hiperactividad del detrusor. Aunque la demostración de ésta última no necesariamente conduce a IU. Para complicar más la idea, los aumentos de presión intrabdominal, en ocasiones, desencadenan hiperactividad del detrusor ${ }^{13}$. En cuanto a que se debe tratar primero si el componente de urgencia o el de esfuerzo, la mayoría de urólogos están de acuerdo en que se debe comenzar por la más predominante. Esta idea coincide con las recomendaciones de la guía de la EUA ${ }^{4}$. Aunque, al no existir criterios de predominancia bien definidos ni clínica ni urodinámicamente, el urólogo utiliza criterios subjetivos de forma individualizada como el impacto sobre calidad de vida, el grado de escape o el grado de la hiperactividad del detrusor. Por otra parte, existe evidencia de que la IUM puede mejorar igualmente con tratamiento antimuscarínico como tras cirugía antiincontinencia de esfuerzo ${ }^{14-15}$. Si fuera así, la necesidad de la urodinámica se cuestionaría. Únicamente con una buena historia clínica sería suficiente. Ya que, cualquiera de los tratamientos lograría bue- nos resultados. Lo cierto es que, la IUM es una de las situaciones clínicas en la que se necesita un mayor conocimiento fisiopatológico. Por lo tanto, es lógico que, ante esta situación confusa, los urólogos respondan conservadoramente. El 85\% trata primero el componente de urgencia al disponer de tratamiento médico sencillo, rápido y reversible. Además, disminuyendo la urgencia se logra mejorar la calidad de vida y, adicionalmente, un porcentaje de mujeres se mantendrían sin operarse.

De acuerdo a las guías consultadas, son altamente recomendables los cambios en el estilo de vida, los ejercicios del suelo pélvico y el entrenamiento vesical durante el tratamiento inicial. Esto sirve para los tres grupos de IU no complicada. Los cambios en el estilo de vida incluyen reducción de peso, abandono del tabaco y modificación de dieta líquida, como por ejemplo reducción de cafeína.

El tratamiento conservador debe ser complementado con apropiada farmacoterapia según el tipo de IU. Los antimuscarínicos para la IUU en el contesto de la vejiga hiperactiva y los inhibidores duales de la recaptación de la serotonina y noradrenalina para la IUE, en caso de que se disponga. Ambas terapias son altamente recomendables $^{4-5}$. Por otra parte, si se detecta deficiencia estrogénica y/o infección del tracto urinario inferior, se debe tratar con terapia sustitutiva o con antimicrobianos. Para posteriormente volver a valorar. Este tratamiento inicial debe mantenerse entre 8-12 semanas antes de reevaluar y antes de mandar al especialista para tratamiento posterior. De acuerdo a las respuestas obtenidas para el tratamiento inicial de la IUU destaca que, sólo el 50\% de los urólogos recomiendan modificar el hábito higiénico dietético. Este dato sorprende, más cuando se trata de una medida simple, barata, conservadora y altamente recomendable. Además, la elevada tasa de respuesta a placebo observada en ensayos clínicos e independientes del tipo de IU, ha puesto de manifiesto que la autodisciplina ejerce un efecto terapéutico por si mismo. Sólo un 17\% recomienda ejercicios del suelo pélvico para la IUU. Lo que contrasta con lo recomendado en las guías. Posiblemente, este dato refleja un error de concepto. Ya que, se cree que es únicamente 
valido para la IUE. El aumento progresivo del porcentaje de urólogos que recomiendan esta medida para la IUM $(48,5 \%)$ y para la IUE $(80,7 \%)$ apoyaría este argumento. Por otra parte, llama la atención que haya un porcentaje minimo, aunque representativo, de urólogos que consideran a estas medidas como tratamiento de segundo nivel.

De acuerdo a la guía de EUA, el uso de antimuscarínicos está indicado en el manejo inicial de la IUU por vejiga hiperactiva y en la IUM. El tratamiento se debe mantener por 8-12 semanas antes de reevaluar y plantear remitir al paciente para tratamiento especializado. Según los resultados obtenidos, prácticamente la totalidad de urólogos cumplen las recomendaciones de la guía en el primer aspecto pero no en cuanto al mantenimiento del tratamiento. Hay que tener en cuenta que, la guía no define el concepto de fallo o paciente no respondedor, ni tampoco el de refractariedad al tratamiento antimuscarínico. Dos conceptos esenciales para el manejo terapéutico de estos pacientes. Esta circunstancia hace que la guía, al menos en este punto, no sea aplicable al ámbito español. Ya que, la mayoría de urólogos españoles suelen mantener el tratamiento por un periodo más largo y además, agotan todos los antimuscarínicos existentes en el mercado antes de considerar que un paciente es refractario al tratamiento. Si se siguieran las recomendaciones de la guía, el porcentaje de pacientes no respondedores o refractarios aumentaría considerablemente. De hecho, llama la atención que, ninguno considera a los antimuscarínicos como tratamiento especializado. Precisamente, porque se trata de agotar todas sus posibilidades terapéuticas antes de plantear un tratamiento más especializado. En este sentido, los resultados obtenidos a la pregunta sobre ¿cuáles son las características del antimuscarínico ideal? son muy interesantes. Sobre todo, porque explican, al menos en parte, el conocido bajo nivel de adherencia de los pacientes a esta medicación a un año vista ${ }^{16-17}$. La práctica totalidad de urólogos consideran a la eficacia como un aspecto muy importante, incluso por delante de la tolerabilidad. Es cierto que se ha producido un gran avance en el grado de tolerabilidad. Ya que, el porcentaje de pacientes que abandonan el tratamiento por efectos adversos es significativamente menor que antes. Pero también, que el urólogo demanda mayor eficacia a estas drogas. En este aspecto, urólogos y pacientes, con su bajo nivel de adherencia, parecen coincidir.

Respecto a la utilización de la neuromodulación, electroestimulación y cirugía, las respuestas coinciden con lo recomendado. Ya que, son terapias a disposición de una minoría de especialistas.

En cuanto a la IUE, aparte de los ejercicios del suelo pélvico, la cirugía es la alternativa más usada. Tanto como tratamiento inicial como en el seguimiento. Aunque no se desvía de lo recomendado por las guías clínicas, destaca que sólo consideran realizar cirugía el 53\%. Porcentaje bajo, que no parece corresponderse con la realidad. Sobre todo, si tenemos en cuenta el gran número de cabestrillos suburetrales implantados. Por otro lado, no se entiende muy bien el uso de otros fármacos. Sobre todo, cuando la duloxetina no esta comercializada en España. Respecto a las respuestas de tratamiento para la IUM coinciden con lo expresado anteriormente.

Es evidente que grado de adherencia a las guías clínicas de IU por los urólogos españoles no es el ideal. El objetivo de este trabajo no era estudiar los motivos de la falta de adherencia. Un análisis de este aspecto esta fuera del alcance de esta discusión. Sin embargo, hay que resaltar que existen múltiples barreras o impedimentos para que los urólogos cumplan con las guías. Hay barreras relacionadas con la ausencia de conocimiento o falta de familiaridad con la guía, con la accesibilidad, con sobrecarga de información científica, etc. Otro tipo de barreras se relaciona con el comportamiento del médico, ya sea por factores dependientes del paciente (preferencias, condicionantes culturales), de la propia guía (recomendaciones complejas o discordantes), ambientales como la ausencia de recursos (tiempo y sistemas de información), la falta de incentivos, o restricciones que emergen desde la organización a la que se pertenece. Por último, hay barreras relacionadas con las actitudes. Entre estas destacan situaciones como la falta de acuerdo con las guías en general o en particular, la falta de expectativas sobre el resultado final, la falta de confianza en la capacidad de llevarlas a cabo, los hábitos y rutinas o la propia experiencia acumulada. Todos factores determinantes del incumplimiento. 
Resumiendo podemos concluir que, las guías clínicas tienen un papel relevante como herramienta de apoyo y mejora en nuestra práctica clínica diaria. El beneficio de su uso es indudable. Sin embargo, es necesaria la realización de programas que mejoren su implantación. Tanto la evaluación de su implantación como el diseño de los programas de mejora necesitan una metodología específica que, por el momento no existe en las guías de ámbito urológico y, en concreto, para la IU. Aún teniendo en cuenta las limitaciones metodológicas de este trabajo, los resultados ponen de manifiesto que la adherencia y cumplimiento de los urólogos a las guías de IU está lejos de lo ideal. Las causas pueden ser muy diversas y dificiles de analizar. En el futuro, es deseable que en el proceso de diseño de guías clínicas se incluya indicadores específicos para poder plantear estudios que analicen el grado de cumplimiento y adherencia de los profesionales a la que va dirigido. Un aspecto esencial de la verdadera utilidad práctica de las guías.

\section{Agradecimientos}

Este trabajo ha sido impulsado y financiado por Almirall Prodesfarma. Además, este trabajo recibió el apoyo técnico de Km Health (GOC Networking).

\section{REFERENCIAS}

1. Stewar WF, Van Rooyen JB, Cundiff GW, Abrams P, Herzog AR, Corey R, et al. Prevalence and burden of overactive bladder in the United States. World J Urol. 2003;20(6):237336.

2. Irwin DE, Milsom I, Reilly K, Hunskaar S, Kopp Z, Herschorn S, Kelleher C, Hampel C, Artibani W, Abrams P. Prevalence of overactive bladder syndrome: European results from the EPIC study. Eur Urol. 2006;50:1306-1315.

3. Mira JJ, Perez-Jover V, Lorenzo S, Aranaz J, Vitaller J. Qualitative research: a valid alternative. Atención Primaria 2004;34(4):161-165.

4. Thüroff J, Abrams P, Andersson KE, Artibani W, ChartierKastler E, Hampel C, van Kerrebroeck Ph. Guidelines on urinary incontinente. In European Association of Urology Guidelines 2007 edition. Ed. EAU Guidelines Office.
5. Abrams P, Andersson KE, Brubaker L, Cardozo L, Cottenden A, Denis L et al. Recommendations of the International Scientific Committee: evaluation and treatment of urinary incontinence, pelvic organ prolapse and faecal incontinence. En P. Abrams, L. Cardozo, S. Koury y A. Wein. $3^{\text {rd }}$ International consultation on incontinence. Edt. Health Publication Ltd. 2005.

6. Castro Díaz D, Garat Barrero JM, Jiménez Cidre M, Martínez-Agulló E, Pena Outeriño XM, Rioja Sanz C et al. Pautas de actuación en la incontinencia urinaria. Consejo de Salud Vesical. Ed. Jarpyo. Madrid, 2001.

7. Krueger RA. El grupo de discusión. Guía práctica para la investigación aplicada. Ed Pirámide. Madrid, 1991.

8. Sand PK. Subjective measures of efficacy: quality of life, patient satisfaction and patient-oriented goals - the search for value. Eur Urol. 2007;(Suppl 6):438-443.

9. Gómez-Doblas, JJ. Implementación de guías clínicas. Rev Esp Cardiol. 2006;59(3):29-35.

10. Morgan. Focus groups as qualitative research. Sage. Bebery Hills, CA, 1988.

11. Seckiner I, Yesilli C, Mungan NA, Aykanat A, Akduman B. Correlations between the ICIQ-SF score and urodynamic findings. Neurourol Urodyn 2007;26(4):492-494.

12. Consenso sobre terminología y conceptos de la función del tracto urinario inferior. El Grupo Español de Urodinámica y de la SINUG. Actas Urol Esp. 2005;29(1):16-30.

13. Lewis JB, Alexander V. O'Connor RC, Guralnick ML. Are there differences between women with urge predominant and stress predominant mixed urinary incontinence? Neurourol and Urodyn 2007;26(2):204-207.

14. Kreder KJ Jr, Brubaker L, Mainprize T. Tolterodine is equaly effective in patients with mixed incontinente and those with urge incontinente alone. BJU International 2003;92(4):418-421.

15. Osman T. Stress incontinente surgery for patients presenting with mixed incontinente and a normal cystometrogramm. BJU Internacional 2003;92(9):964-968.

16. Haab F, Cardozo L, Chapple C, Ridder AM, Solifenacin Study Group. Long term open-label solifenacin treatment associated with persintence with therapy in patients with overactive bladder syndrome. Eur Urol. 2005;47(3):376-384.

17. Hampel $\mathrm{Ch}$. Long term management of overactive bladder with antimuscarinic agents. Eur Urol. 2007;(Supple 6): 432-437.

Correspondencia autor: Dr. J.L. Ruiz Cerdá

Servicio de Urología. Hospital Universitario La Fe

Avda. Campanar 21 - 46009 Valencia. Tel.: 963862700

E-mail autor: Jose.L.Ruiz @uv.es

Información artículo: Original - Incontinencia urinaria femenina

Trabajo recibido: abril 2007

Trabajo aceptado: junio 2007 